\title{
The DRG-Based Hospital Prospective Payment System in Greece: An Assessment of the Reimbursement Rates Using Clinical Severity Classification
}

\author{
A. Vozikis ${ }^{1}{ }^{*}$, S. Xesfingi ${ }^{1}$, E. Moustaferi ${ }^{1}$, T. Balbouzis ${ }^{2}$, T. Rigatos ${ }^{2}$ \\ ${ }^{1}$ Laboratory of Health Economics and Management, Department of Economics, University of Piraeus, Piraeus, Greece \\ ${ }^{2}$ Department of Strategic Planning, National Organization for Provision of Healthcare Services (EOPYY), Athens, Greece \\ Email: *avozik@unipi.gr
}

How to cite this paper: Vozikis, A., Xesfingi, S., Moustaferi, E., Balbouzis, T. and Rigatos, T. (2016) The DRG-Based Hospital Prospective Payment System in Greece: An Assessment of the Reimbursement Rates Using Clinical Severity Classification. Modern Economy, 7, 1584-1600. http://dx.doi.org/10.4236/me.2016.713141

Received: August 25, 2016

Accepted: November 28, 2016

Published: December 1, 2016

Copyright $\odot 2016$ by authors and Scientific Research Publishing Inc. This work is licensed under the Creative Commons Attribution International License (CC BY 4.0).

http://creativecommons.org/licenses/by/4.0/

\section{(c) (i) Open Access}

\begin{abstract}
Introduction: Diagnosis Related Groups (DRGs) are used throughout the health care industry to address issues of cost, effectiveness and quality of care. Nonetheless, many have argued that the DRGs do not adequately adjust for patient severity and this prospective payment system leads to reimbursement inequities. Objective: The purpose of this study is first, to explore the relationship between KEN-DRGs reimbursement rates (along with other controls) and clinical severity classification and second, to draw hospital reimbursement policy implications. Methods: The present study is a retrospective observational study, undertaken in a very large number of hospitalization cases and conducted exclusively by examining reimbursement administrative data collected from the e-DAPY database of National Organization for Provision of Healthcare Services (EOPYY) in Greece. The database provided contained all impatient hospitalizations classified for every single KEN-DRG code and for every MDC (aggregated) for the time period January to June 2013 (first semester of 2013). Results: Our results demonstrate that though there is a strong association between reimbursement rates and clinical severity, there are several KEN-DRG codes that are over-(under-) reimbursed, taking in consideration their clinical severity class. Conclusion: Because DRGs systems are crucial in measuring relative efficiency among hospitals, as well as driving ongoing reimbursement, they should incorporate and establish the severity level for every admission, so as to determine whether case mix growth is associated with real costs or with coding and documentation improvements at the hospital.
\end{abstract}




\section{Keywords}

DRGs, Prospective Payment Systems, Hospital Reimbursement, Case-Mix, Greek National Health System

\section{Introduction}

Diagnosis Related Groups (DRGs) are used throughout the health care industry to address issues of cost, effectiveness and quality of care. The design and development of the DRGs began in the late ' 60 s at Yale University. The initial motivation for developing the DRGs was to create an effective framework for monitoring the quality of care and the utilization of services in a hospital setting [1]. The first large-scale application of the DRGs was in the late "70s in the USA and specifically in the State of New Jersey [2], which used DRGs as the basis of a prospective payment system in which hospitals were reimbursed a fixed DRG specific amount for each patient treated. In 1982, a modification of Medicare hospital reimbursement limits was made to include case mix (number and type of cases treated in a hospital) adjustment based on DRGs, as a recognition of the fundamental role which a hospital's case mix plays in determining its costs [3]. In 1983, USA proceeded to a national DRG-based hospital prospective payment system for all Medicare patients [1] [4].

Nonetheless, many have argued that the DRGs do not adequately adjust for patient severity and this prospective payment system leads to reimbursement inequities [4] [5]. To respond to these criticisms, the Centers for Medicare and Medicaid Services (CMS) developed the CMS severity-adjusted DRGs (SDRGs), by refining the existing DRG structure and better identifying patients with different resource needs and outcomes. However, the SDRGs, like the CMS DRGs, suffered from being targeted to the over sixty-five year old population and from serious conceptual limitations in certain key areas (neonatal care, etc.), that reduced SDRG system's ability to uniformly predict severity of illness [5] [6].

In 1987 the original DRG system was revised into an All-Patient DRG (AP-DRG) system. The AP-DRGs are an expansion of the basic DRGs to be more representative of non-Medicare populations [7]. In 2003, the All-Patient-Refined DRG (APR-DRG) system was introduced, shifting the system's focus to patient characteristics including adjustments for severity of the condition and co-morbidities [1] [5] [8]. The APS-DRGs have a simple, explicit, and easily understood structure and they are based upon the SDRG, but address the limitations discussed above [9]-[11].

A number of studies indicate the importance of clinical severity as a significant component of an equitable prospective payment system, therefore and the importance of APS-DRGs [7] [12] [13].

Many other countries implemented Diagnosis Related Groups (DRGs) or similar grouping systems as instruments for hospital reimbursement [14] [15]. Australia was one of the first countries that followed the USA paradigm, and adapted its own specific 
AR-DRG classification based upon 23 major diagnostic categories (MDC) [16]. Nordic countries [17] (Norway, Sweden, Finland and Denmark) introduced the Nordic DRGs (Nord DRGs) based on a refined DRG from Health Care Financing Administration (HFA-DRGs) [18], Estonia [19], has introduced and gradually implemented (50\% of case cost) the Nord DRGs since 2003. Iceland [14] has been using the Nord DRGs on a trial basis in Landspitali University Hospital since 2001. Portugal in 1989 introduced "Foltha de Admissao e Alta", which is a basic information system that is now used by all NHS hospitals [14] [17]. Ireland has adapted Portugal's methodology and in 1993 introduced a similar method of hospital reimbursement based on DRGs [17]. Since 2002 the $15 \%$ of hospitals budget in Portugal is covered by case-mix and $85 \%$ based on historical cost. Italy has introduced in 1999 prospective payments for hospitals based on DRGs [14]. The Italian tariff system is based on DRGs to constrain budgets [15] [20]. In 2003 German Hospitals introduced a payment system (which became mandatory from 2004) based on DRGs [21] [22], based on an adaptation and further development of the refined Australian AR-DRGs [15]. French DRGs were initially developed in 1986. DRG-like hospital payment system called "Tarification á l' Activite" was introduced in 2004-2005 as a basic health care reform [14]. The Greek NHS on the other hand, up until recently was financed on the basis of a complex and anachronistic model such as fixed budgets, fixed rates per admission or per diem rates based on the number of bed days, which couldn't effectively support and respond adequately to the developments and needs of modern health care system [23]. For this, in 2011 it was decided the establishment of a Committee with main objective to explore the feasibility of implementing the DRGs as a system of prospective financing the Greek NHS Hospitals and to establish the Greek Medical Procedure coding [24] [25].

The Australian Refined DRGs (AR-DRGs) Classification system, which had been already implemented in Germany the last nine years [15] [22], was chosen as the most appropriate for application in Greece, after extensive review of the literature and thorough evaluation of global experience in application of different reimbursement systems based on the DRGs [17].

Because of the urgency to reform the health care system, and reimburse hospitals at their real health services production cost and hence to eliminate structural deficits, the Committee evaluated the option to use the cost weights of the AR-DRGs [23] [24], as well as the Australian AR-DRGs [23] and implement them in the Greek DRG system as an indirect solution to the issue of cost assessment. Since the end of 2011 (with an adjustment-correction in coding, naming, costing and average length of stay (ALoS) as of February 2012) the implementation of the Greek version of DRG's (hereinafter referred to as "KEN-DRGs") has been decided by the Greek authorities, in order to improve the efficiency of the health hospital sector and to contain the cost of care [24] [25].

The purpose of this study is first, to explore the relationship between KEN-DRGs reimbursement rates (along with other controls) and clinical severity classification and second, to draw policy implications. Our results demonstrate that though there is a strong association between reimbursement rates and clinical severity, there are several 
KEN-DRG codes that are over-(under-) reimbursed, taking in consideration their clinical severity class.

\section{Data and Methodology}

Aggregate hospitalizations data were collected from the e-DAPY database of National Organization for Provision of Healthcare Services (EOPYY) in Greece. EOPYY is the largest social security fund (SSF) covering more than $95 \%$ of the insured population, and was founded in 2012 from the merge of various individual SSFs. In the first months of 2012 the four biggest SSFs (IKA, OAEE, OGA, OPAD) formed EOPYY while by November 2012 the majority of the remaining funds were also incorporated into EOPYY. EOPYY provides health insurance under a single benefits package for the $95 \%$ of Greek population, either through a network of contracted physicians, public and private hospitals and diagnostic centres or through the health centers of PEDY.

In the present study, a database was provided for the time period January to June 2013 (first semester of 2013) containing all impatient hospitalizations classified for every single KEN-DRG code and for every MDC (aggregated). The present study is a retrospective observational study, undertaken in a very large number of hospitalization cases and conducted exclusively by examining reimbursement administrative data. Data regarding the patient's age and gender, diagnosis, number of hospitalizations, percentage of co-payment and patient's unique social security number were not provided. The data provided from the EOPYY for the purpose of this study, where aggregated, anonymized in respect of any patients' identification. Permission for use of anonymized data was obtained by the administration of EOPYY, in accordance to the national legislation on the Protection of Individuals with regards to the Processing of Personal Data. The study has been approved by the Scientific Committee of the University of Piraeus.

The provided database contained exclusively the following data:

MDC code, MDC name, KEN-DRG code, KEN-DRG name, ALoS, $\mathrm{N}$ of cases, Reimbursement rate (in $€$ ), Total reimbursed amount (in $€$ )

(Predetermined ALoS and Reimbursement rate in the Greek NHS Hospitals for the selected KEN-DRGs, were derived from Gazette 946/27/03/2012).

\subsection{Empirical Analysis}

All variables, except to Clinical Severity, are continuous. We have clustered all of them in three classes with respect to their frequencies. The third percentile is used for all variables in order to cut off the $33.33 \%$ of the data values when they are sorted in ascending order. More specifically, the variable Cost (Total reimbursed amount in $€$ ) takes the value 1 for $€ 136$ - €1981, 2 for $€ 1988$ - €3908 and 3 for $€ 4000$ - €55,695; the variable EconComp (Reimbursement rate in $€$ ) takes the value 1 for $0.14-2.12$, 2 for $2.14-4.14$ and 3 for 4.27 - 55.64; the variable ALS (Average Length of Stay) takes the value 1 for 1 - 3 days, 2 for 4 - 8 days and 3 for 9 - 85 days. And finally, the variable Clin Sev (Clinical Severity) takes the values 1, 2 and 3 for low, medium and high clinical severity, respectively. 
Table 1 below presents the summary statistics of our data.

As Table 1 shows, there are 24 Major Diagnosis Groups (MDC), but the number of subcategories included is not equal for all of them. The minimum DRG Total reimbursed amount is $€ 136$, but there are several DRGs with their Total reimbursed amount exceeding $€ 55,000$, affecting the average Total reimbursed amount that is higher than $€ 2,600$. The same is also documented for the variable Econ Comp (Reimbursement rate in $€$ ). The majority of cases are of medium or high clinical severity and the average length of stay is nearly 8 days.

Table 2, below, presents the correlations across all variables.

Table 2 further demonstrates that there is a strong correlation between variable Econ Comp (Reimbursement rate in $€$ ) and Cost (Total reimbursed amount in $€$ ), as the Pearson correlation coefficient is larger than 0.9. This relies on the fact that the Total reimbursed amount of each DRG is fully based on its Reimbursement rate; therefore, there is no reason for the two variables to be both included in the same model. A weaker correlation is demonstrated between Cost and ALS (Average Length of Stay) (0.7), but the most interesting is the correlation demonstrated between Cost and ClinSev (Clinical Severity) (smallest Pearson correlation coefficient; 0.3).

Pie charts, next, provide more details about the distribution of the aforementioned variables.

As shown by Figure 1, there is an almost perfect match between EconComp (Reimbursement rate in $€$ ) and Cost (Total reimbursed amount in $€$ ) distributions. With respect to Average Length of Stay, an equal distribution is documented since the percentages for the classes 1, 2 and 3 are 35\%,34\% and 31\%, respectively. The largest "disagreement" is demonstrated with respect to Clinical Severity. Since the $49 \%$ of the DRGs is considered to be of high clinical severity, someone would expect that those cases

Table 1. Descriptive statistics.

\begin{tabular}{cccccc}
\hline Variables & Observations & Mean & Std. Dev. & Min & Max \\
\hline DRGs & 653 & 11.741 & 6.221 & 1 & 24 \\
Cost & 653 & $2,614.049$ & $4,606.065$ & 136 & 55,695 \\
ClinSev & 653 & 2.283 & 0.788 & 1 & 3 \\
EconComp & 653 & 2.696 & 4.080 & 0.14 & 55.639 \\
ALS & 653 & 7.832 & 8.348 & 1 & 85 \\
\hline
\end{tabular}

Table 2. Correlations.

\begin{tabular}{ccccc}
\hline Variables & Cost & ClinSev & EconComp & ALS \\
\hline Cost & 1.000 & & & \\
ClinSev & $0.337^{\star}$ & 1.000 & & \\
EconComp & $0.933^{\star}$ & $0.369^{*}$ & 1.000 & 1.000 \\
ALS & $0.724^{\star}$ & $0.445^{\star}$ & $0.812^{\star}$ & \\
\hline
\end{tabular}

Note: $\left({ }^{*}\right)$ indicates significance at $5 \%$ level of significance. 


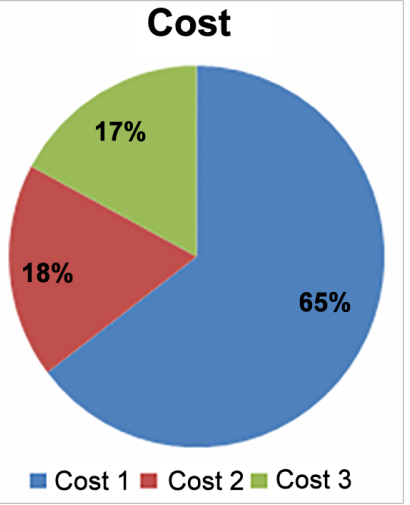

Clinical Severity

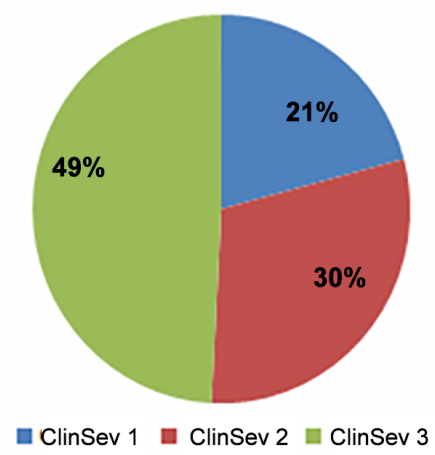

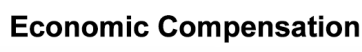

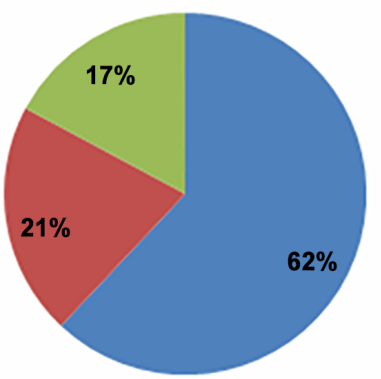

EconComp 1 EconComp 2 EconComp 3

Average Length of Stay

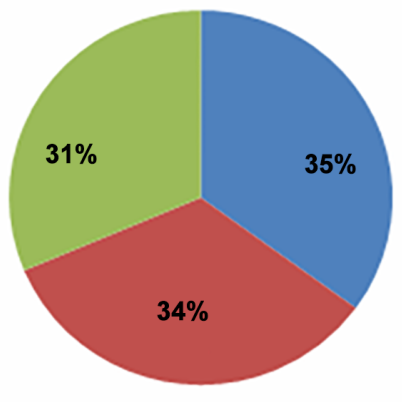

ALS 1 ALS 2 ALS 3

Figure 1. Distribution of all variables.

would lead to an extensive length of stay and therefore to a higher reimbursement rate. Although the $31 \%$ of the DRGs are in class 3 for the Average Length of Stay, this is not the case for Econ Comp (Reimbursement rate in $€$ ) as well (only 17\% of the cases are in the class of higher reimbursement rate).

We are trying to explore furthermore the relation between Reimbursement rate and Clinical Severity. Pie charts, above, and Table 2 with Pearson correlation coefficients demonstrate that there is a considerable deviation since there are several DRGs that do not belong in the same class both of Reimbursement rate and Clinical Severity.

Histogram, below, clearly presents the aforementioned deviation.

Histogram above presents (Figure 2) the relation between clinical severity (horizontal axis) and reimbursement rate in $€$ (here mentioned as Economic Compensation, Econ Comp). For example, the first column shows that among the DRGs belonging to the first cluster of clinical severity (low clinical severity), $2 \%$ of them are compensated with a medium reimbursement rate. More specifically, only $18.5 \%$ of the DRGs belonging to the medium clinical severity cluster (column 2) are also compensated with a medium reimbursement rate, while $78 \%$ and $3.5 \%$ of them are compensated with a lower and higher reimbursement rate, respectively. Finally, as column 3 demonstrates, only $33 \%$ of the DRGs that are considered to be of high clinical severity are compensated with a high reimbursement rate, while $30 \%$ and $37 \%$ of them are compensated with a medium and lower reimbursement rate, respectively. 
The cross tabulation below (Table 3), where the Histogram 3 is transformed in frequencies, presents how many of the DRGs are clustered in each class of Reimbursement rate and Clinical Severity.

Table 5 shows that for most DRGs, Reimbursement rate does not match with the degree of Clinical Severity In other words, in many cases, DRGs receive lower Reimbursement rate than they should have got according to their clinical severity status. For instance, DRGs with "high" clinical severity are 321 and only 105 (32.7\%) are reimbursed with "high" rate. Same finding holds for the DRGs with "medium" clinical severity. For example, 153 out of 196 DRGs exhibit lower Reimbursement rate for their clinical severity. However, the majority of the DRGs that exhibit "low" clinical severity, which consist the $20.8 \%$ of our sample, gets also "low" Reimbursement rate. In this case, there is a match in Reimbursement rate and clinical severity. Overall, $56.5 \%$ of the DRGs show Reimbursement rate lower than their corresponding clinical severity, while economic compensation and clinical severity exactly match in $42 \%$ of the DRGs. That leaves $1.5 \%$ of DRGs where Reimbursement rate is higher than their clinical severity.

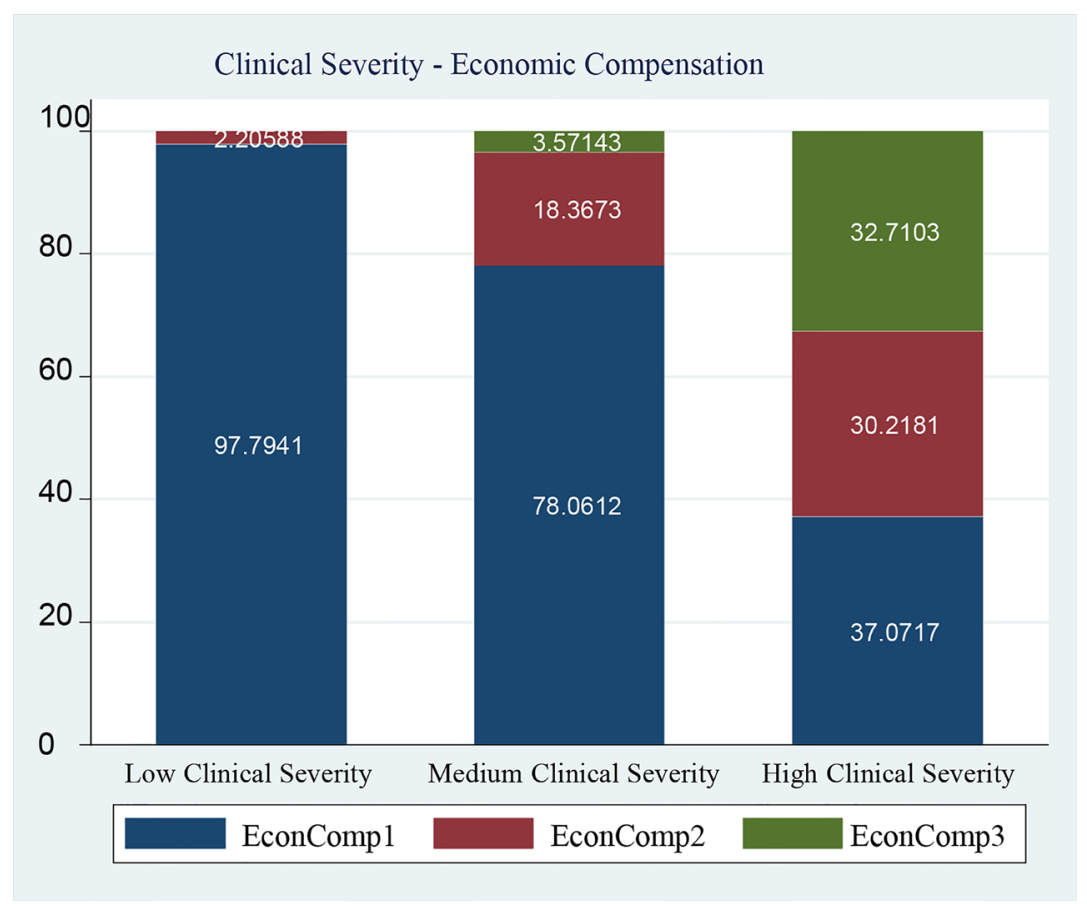

Figure 2. Relation between reimbursement rate and clinical severity.

Table 3. Cross tabulation of EconComp and ClinSev.

\begin{tabular}{ccccc}
\hline & \multicolumn{5}{c}{ Economic Compensation } \\
\hline Clinical Severity & 1 & 2 & 3 & Total \\
Low & 133 & 3 & 0 & 136 \\
Medium & 153 & 36 & 7 & 196 \\
High & 119 & 97 & 105 & 321 \\
Total & 405 & 136 & 112 & 653 \\
\hline
\end{tabular}


Although this result does not seem very important with respect to the total budget spent on DRGs, there are several categories that vary in number of cases treated and their corresponding Total reimbursed amount in $€$.

Table 4 presents the DRGs that seem to have a small or large deviation between the number of cases reported and their clinical severity.

As Table 4 indicates, the number of cases treated plays also an important role. It appears that DRGs with higher clinical severity do not seem to burden the total budget compared to DRGs with medium and low clinical severity.

Next, Table 5 presents the deviation documented between Total reimbursed amount in $€$ and clinical severity.

Table 4. Case mix with respect to clinical severity.

\begin{tabular}{|c|c|c|c|c|c|c|c|c|}
\hline \multicolumn{3}{|c|}{ A. Low clinical severity } & \multicolumn{3}{|c|}{ B. Medium clinical severity } & \multicolumn{3}{|c|}{ C. High clinical severity } \\
\hline DRG & $\begin{array}{l}\text { Number } \\
\text { of cases }\end{array}$ & $\begin{array}{l}\% \text { total } \\
\text { budget }\end{array}$ & DRG & $\begin{array}{l}\text { Number } \\
\text { of cases }\end{array}$ & $\begin{array}{l}\% \text { total } \\
\text { budget }\end{array}$ & DRG & $\begin{array}{l}\text { Number } \\
\text { of cases }\end{array}$ & $\begin{array}{l}\% \text { total } \\
\text { budget }\end{array}$ \\
\hline $\mathrm{T} 27 \mathrm{X}$ & 11,176 & $0.0358 \%$ & L01X & 8931 & $0.0669 \%$ & D06A & 842 & $0.0087 \%$ \\
\hline $\mathrm{O} 16 \mathrm{~A}$ & 8209 & $0.0286 \%$ & L02X & 5929 & $0.0355 \%$ & $\mathrm{~A} 22 \mathrm{Mb}$ & 778 & $0.0061 \%$ \\
\hline $\mathrm{C} 10 \mathrm{~A}$ & 5762 & $0.0127 \%$ & W10A & 1734 & $0.0077 \%$ & TH06X & 678 & $0.0069 \%$ \\
\hline L05A & 3124 & $0.0047 \%$ & P10X & 1679 & $0.0109 \%$ & M03X & 659 & $0.0148 \%$ \\
\hline $\mathrm{C} 08 \mathrm{~A}$ & 2643 & $0.0119 \%$ & H08X & 1339 & $0.0109 \%$ & S22X & 604 & $0.0029 \%$ \\
\hline C09A & 1939 & $0.0058 \%$ & W11A & 1336 & $0.0048 \%$ & $\mathrm{~A} 22 \mathrm{Ma}$ & 564 & $0.0074 \%$ \\
\hline D27X & 1555 & $0.0017 \%$ & Y07X & 1303 & $0.0063 \%$ & W06A & 563 & $0.0034 \%$ \\
\hline $\mathrm{K} 32 \mathrm{~A}$ & 1283 & $0.0048 \%$ & L26A & 1300 & $0.0019 \%$ & M04A & 561 & $0.0168 \%$ \\
\hline Y22A & 1278 & $0.0046 \%$ & D07A & 1130 & $0.0051 \%$ & N03X & 495 & $0.0096 \%$ \\
\hline D09A & 997 & $0.0047 \%$ & $\mathrm{~A} 22 \mathrm{X}$ & 949 & $0.0041 \%$ & $\mathrm{~K} 46 \mathrm{X}$ & 481 & $0.0016 \%$ \\
\hline \multicolumn{2}{|c|}{ Total } & $0.1153 \%$ & \multicolumn{2}{|c|}{ Total } & $0.1541 \%$ & \multicolumn{2}{|c|}{ Total } & $0.0782 \%$ \\
\hline
\end{tabular}

Table 5. Total reimbursed amount in $€$ with respect to clinical severity.

\begin{tabular}{ccccccccc}
\hline \multicolumn{2}{c}{ A. Low clinical severity } & \multicolumn{2}{c}{ B. Medium clinical severity } & \multicolumn{2}{c}{ C. High clinical severity } \\
\hline DRG & Cost (in $€$ ) $\begin{array}{r}\text { \% total } \\
\text { budget }\end{array}$ & DRG & Cost in $€$ ) & $\begin{array}{r}\% \text { total } \\
\text { budget }\end{array}$ & DRG & Cost (in $€$ ) & $\begin{array}{l}\% \text { total } \\
\text { budget }\end{array}$ \\
\hline M02X & 3000 & $0.0027 \%$ & K01X & 11,291 & $0.0021 \%$ & E10A & 55,695 & $0.0021 \%$ \\
K17X & 2227 & $0.0021 \%$ & D01X & 5865 & $0.0001 \%$ & E05A & 34,000 & $0.0051 \%$ \\
M73M & 2044 & $0.0010 \%$ & N42Ma & 5277 & $0.0001 \%$ & T22A & 23,575 & $0.0002 \%$ \\
D20X & 1471 & $0.0008 \%$ & T06X & 4582 & $0.0001 \%$ & E06A & 20,000 & $0.0048 \%$ \\
P04X & 1434 & $0.0007 \%$ & D14A & 3834 & $0.0053 \%$ & T02A & 17,418 & $0.0031 \%$ \\
W04X & 1383 & $0.0009 \%$ & A20M & 3360 & $0.0001 \%$ & K01M & 12,500 & $0.0001 \%$ \\
D29X & 1377 & $0.0006 \%$ & J01A & 3199 & $0.0003 \%$ & E06Xa & 12,000 & $0.0025 \%$ \\
N23A & 1326 & $0.0025 \%$ & M68Ma & 3069 & $0.0005 \%$ & K03M & 11,277 & $0.0040 \%$ \\
N42X & 1288 & $0.0001 \%$ & P04Ma & 3028 & $0.0007 \%$ & M06A & 9431 & $0.0001 \%$ \\
B06M & 1212 & $0.0001 \%$ & K19A & 2903 & $0.0015 \%$ & M32Ma & 9083 & $0.0001 \%$ \\
\multicolumn{2}{c}{ Total } & $0.0115 \%$ & \multicolumn{2}{c}{ Total } & $0.0108 \%$ & & Total & $0.0221 \%$ \\
\hline
\end{tabular}


Although the number of cases is small for the DRGs with high clinical severity (panel C, of Table 5), their contribution to the total budget is high.

We are interested in cases, where some DRGs receive higher Reimbursement rate than their clinical severity indicates.

Finally, Table 6 presents deviations (large vs. small) between Reimbursement rate and clinical severity, showing also a reference to the Total reimbursed amount of each DRG of interest as a \% of Total reimbursed amount (the Codes description of Table 8, are presented in the Annex)

As Table 6 shows, for the majority of the DRGs the Reimbursement rate is higher than the corresponding clinical severity (right part of Table 6), although the deviation documented is small for more than $65 \%$ of the categories. For example, there are three DRGs of "low" clinical severity but are reimbursed as if they were of "medium" severity (M02X, M73M and N23A). Additionally, there are seven DRGs of "medium" clinical

Table 6. Deviation between reimbursement rate and clinical severity.

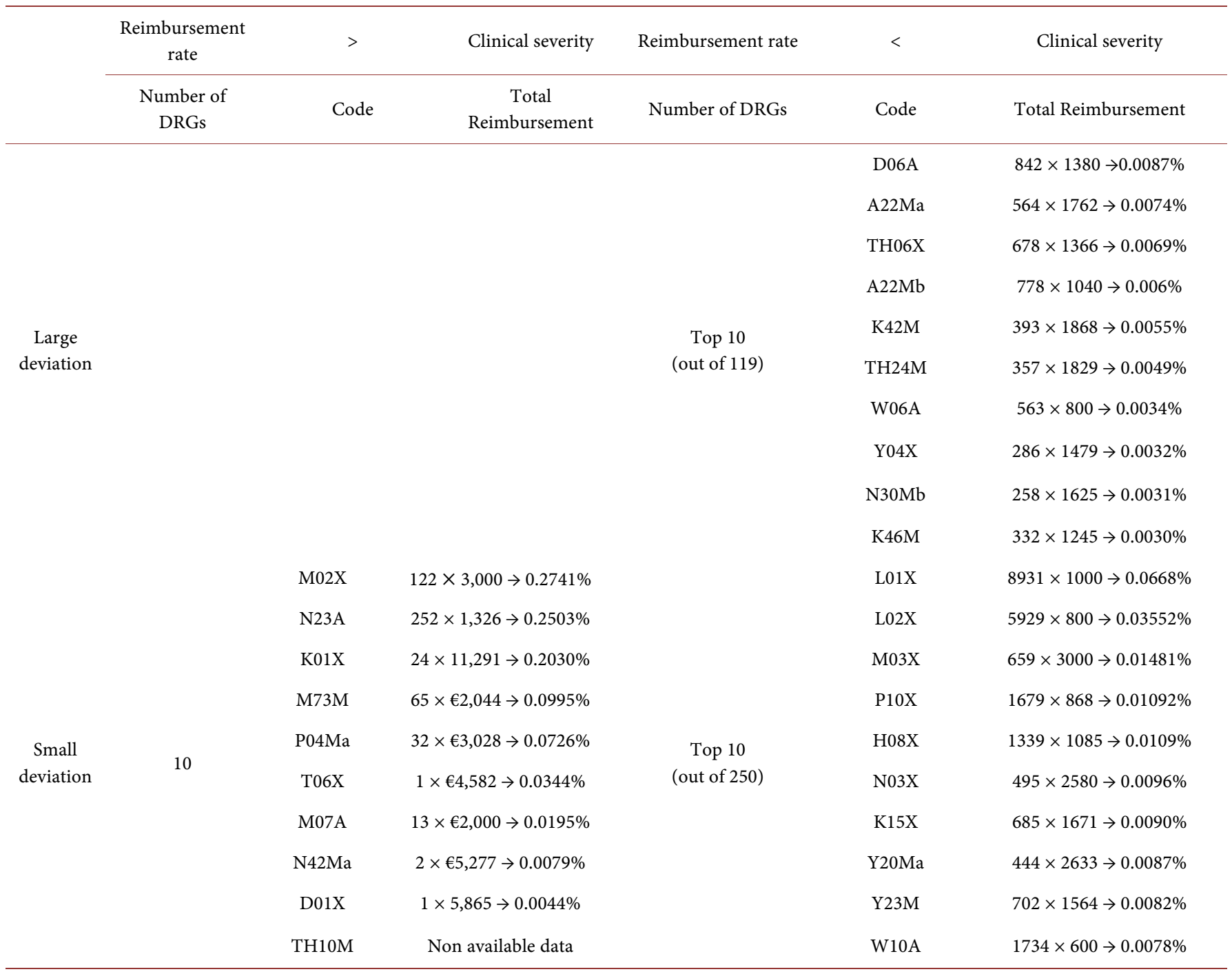


severity and are rated as if they were of "high" clinical severity (D01X, K01X, M07A, N42Ma, P04M, T06X and TH10M). The same findings are presented as well in Table 3. If we want to put some numbers behind, almost $1 \%(0.97 \%)$ of the budget goes to DRGs where the Reimbursement rate is actually higher than the clinical severity.

\subsection{Estimated Model}

We now turn into quantifying the effect of clinical severity on Reimbursement rate setting. In order to pull outlying data from a positively skewed distribution closer to the bulk of the data in a quest to have the variable to be normally distributed, we use logarithmic transformation. A linear relationship is hypothesized between a log-transformed outcome variable, here Econ Comp (Reimbursement rate in $€$ ), and a group of predicted variables.

$$
\log (Y i)=\beta 0+\beta 1 \text { ClinSev } 2+\beta 2 \text { ClinSev } 3+\beta 3 A L S 2+\beta 4 A L S 3+\varepsilon i
$$

where $Y i$ is the dependent variable of Econ Comp, $\beta 0$ is the intercept, ClinSev2 is a dummy that takes the value 1 if the clinical severity of the DRG is medium and 0 otherwise; ClinSev3 is a dummy that takes the value 1 if the clinical severity of the DRG is high and 0 otherwise; ALS2 is a dummy that takes the value 1 if the average length of stay is between 4 - 8 days and 0 otherwise; ALS3 is a dummy that takes the value 1 if the average length of stay is larger than 8 days and 0 otherwise.

Table 7 reports the regression coefficients for the log-transformed variables of Econ Comp without and with DRGs dummies.

We assume that Econ Comp is log-normal conditional on all the covariates and since this is just an ordinary least squares regression, we can easily interpret the regression coefficient, say $\beta 1$, as the expected change in log (Econ Comp) with respect to a oneunit increase in X1 holding all other variables at any fixed values. If we want to interpret the changes of Reimbursement rate with respect to one-unit increase in $\mathrm{X} 1$, we have to calculate the exponentiated values of the regression coefficients of previous Table.

Next, Table 8 demonstrates this interpretation.

Table 7. Regression estimates (dependent variable: log of EconComp).

\begin{tabular}{ccc}
\hline Regression coefficients & LogEconComp Without & LogEconComp w/DRGs dummies \\
\hline ClinSev $_{2}$ & $0.393^{* * *}(0.061)$ & $0.392^{* * *}(0.061)$ \\
ClinSev $_{3}$ & $0.676^{* * *}(0.068)$ & $0.602^{* * *}(0.071)$ \\
$\mathrm{ALS}_{2}$ & $0.768^{* * *}(0.059)$ & $0.797^{* * *}(0.058)$ \\
$\mathrm{ALS}_{3}$ & $1.622^{* * *}(0.071)$ & $1.596^{* * *}(0.068)$ \\
DRGs dummies & No & Yes \\
Constant & $-0.770^{* * *}(0.041)$ & $-0.735^{* * *}(0.044)$ \\
Observations & 653 & 653 \\
$\mathrm{R}^{2}$ & 0.6860 & 0.7472 \\
$\mathrm{~F}$ & 351.05 & 359.83 \\
\hline
\end{tabular}


Table 8. Interpretation.

\begin{tabular}{ccc}
\hline & EconComp Without & EconComp w/DRGs dummies \\
\hline ClinSev $_{2}$ & 1.48 & 1.48 \\
ClinSev $_{3}$ & 1.96 & 1.82 \\
$\mathrm{ALS}_{2}$ & 2.15 & 2.22 \\
$\mathrm{ALS}_{3}$ & 5.06 & 4.93 \\
DRGs dummies & No & Yes \\
Constant & 0.46 & 0.48 \\
\hline
\end{tabular}

Column 1 presents the estimates EconComp without the DRG dummies. One can read these coefficients as follows: the Reimbursement rate of a DRG will increase by $48 \%$ [ $=(1.48-1) \times 100 \%]$ if its clinical severity is 2 with respect to 1 . Further, the Reimbursement rate will increase by $96 \%$ if the clinical severity of the DRG is 3 instead of 1 . The specific DRGs that affect the Total reimbursed amount are presented in the left part of Table 6. Average length of stay seems to have a greater impact on Reimbursement rate. Particularly, if the average length of stay is 2 and 3 instead of 1 , the Reimbursement rate of the DRG will increase by $115 \%$ and $406 \%$, respectively. The results do not vary significantly even if someone takes into account the variability among different DRGs (Column 2).

\section{Discussion}

The contribution of the present study is twofold: first to empirically assess the relationship between DRGs reimbursement rates and clinical severity classification. Thus far, just few studies performed any type of empirical analysis, but they were limited only in reporting the economic cost of the case mix [12] [13] [25] [26].

Specifically the survey of Horn et al. [12] demonstrated that Severity of Illness/procedure-adjusted DRGs are much more homogeneous than DRGs themselves: overall DRGs explain the $28 \%$ of variability in resource use, while Severity-adjusted DRGs explain the $61 \%$. Therefore a Severity-adjusted DRG payment system appears to be much more equitable than a pure DRG prospective payment system. Furthermore the Severity of Illness Index in the same study explained more than $10 \%$ of the variability in resource use in $94 \%$ of the DRGs, which contained $97 \%$ of the patients, and more than $50 \%$ of the variability in resource use in $36 \%$ of the DRGs, which contained $24 \%$ of the patients.

Respectively results were demonstrated by the Averill et al. [13] Severity of Illness evaluation study. Severity of illness was measured using the Computerized Severity Index (CSI) and was found to be a significant determinant of hospital cost in 76 DRGs that accounted for $41.4 \%$ of the total direct hospital patient care costs and $27 \%$ of the patients. The addition of CSI severity levels to the 76 DRGs reduced the coefficient of variation of cost in these DRGs by $17.4 \%$ and improved the overall reduction in variance of cost within the 76 DRGs by $38.2 \%$. The same study also demonstrated that the 
change in total hospital payments due to the addition of severity for the 76 DRGs varied from a positive $5.71 \%$ to a negative $5.48 \%$.

Another study [27] comparing various DRG-models to use for internal or external benchmarking, concluded that 1) more than 60 percent of the variation in cost and more than 47 percent of the variation in LOS can be explained by the APR-DRGs model 2) the increase in severity of illness level is not attributable to a higher case mix in Belgian hospitals. Using simple regression, they found that there is a significant relation between the average number of diagnoses coded on a hospital level and the fraction of inpatient stays with severity level Minor and 3) not only can the average number of diagnoses coded have an impact on the performance of a hospital when using severityadjusted classification systems, the overall coding quality and accuracy have an impact [28] [29] [30].

A study by Medpac [31] analyzes how CMS annually reviews the MS-DRG definitions to ensure that each group continues to include cases with clinically similar conditions requiring comparable amounts of inpatient resources. When the review shows that subsets of clinically similar cases within an MS-DRG consume significantly different amounts of resources, CMS often reassigns them to a different MS-DRG with comparable resource use or creates a new MS-DRG.

In its recent report KCE propose a framework for a reform of the payment system of hospital care in Belgium [32]. The main rationale for reforming hospital payment systems is to change behaviour by creating incentives for e.g. higher quality or lower costs. However, these "theoretical" incentives have to be confronted with real-world behaviour to evaluate the merits and shortcomings of each payment system.

In its previous report [33] KCE performing a comparative analysis of hospital care payments in five countries, concludes that payment by DRGs does not directly provide incentives for health promotion, disease prevention, self-management, coordination of care between multiple providers and settings.

Other studies [34] [35] found that no severity measure explained differences among hospitals in average LOS. Other patient characteristics, practice patterns, or institutional factors may cause the wide differences across hospitals in LOS.

Another study [36] found that the calculation of nursing cost by stay and by DRG for hospital financing purposes must be calculated on nursing activity data, that more reflect resources used in wards, and not on LOS data.

Finally, a study performed in a neonatal unit [37], found that, it is doubtful that existing models of hospital reimbursement incentivize improvements in quality of care that might reduce disease complexity and complication rates. And concludes that it needs to continually adjust what it rewards and discourages on the basis of feedback from patients and care providers.

\section{Conclusions-Proposals}

Because coding is crucial in measuring relative efficiency among hospitals as well as driving ongoing reimbursement, we will need a better understanding of hospitals' coding 
practices for any severity-adjusted grouper. Because DRGs make extensive use of diagnosis and procedure codes should incorporate and establish the severity level for an admission, so as to determine whether case mix growth is associated with real costs or with coding and documentation improvements at the hospital. Consequently, we are proposing a policy for requiring each hospital to submit an annual case mix audit based on a random sample of its cases.

Furtherer more we suggest the auditing of current medical records not only for coding accuracy but for the adequacy of underlying clinical documentation by the medical staff. The establishment of a clinical documentation management program in collaboration with the medical staff is a way to ensure most accurate and complete documentation of each diagnosis and procedure for every patient. As well as auditing establishment of documentation processes to ensure corporate compliance, measure process adherence, and quantify program success. Also it is crucial for the hospitals leadership to understand that the physicians' involvement in management will increase their responsibility and accountability.

\section{Acknowledgements}

We are grateful to Claire Economidou for providing useful comments. This paper was supported by the Laboratory of Health Economics and Management (Lab HEM), Department of Economics, University of Piraeus, Piraeus, Greece.

\section{References}

[1] 3M (2003) All Patient Refined Diagnosis Related Groups (APR-DRGs), Version 20.0, Methodology Overview, 2003, released by 3M Health Information Systems.

https://www.hcup-us.ahrq.gov

[2] Hsiao, W.C., Sapolsky, H.M., Dunn, D.L. and Weiner, S.L. (1986) Lessons of the New Jersey DRG Payment System. Health Affairs, 5, 32-45. http://dx.doi.org/10.1377/hlthaff.5.2.32

[3] Fetter, R.B., Shin, Y., Freeman, J.L., Averill, R.F. and Thompson, J.D. (1980) Case Mix Definition by Diagnosis-Related Groups. Med Care, 18, 1-53.

[4] Mayes, R. (2007) The Origins, Development, and Passage of Medicare's Revolutionary Prospective Payment System. Journal of History of Medicine and Allied Sciences, 62, 21-55. http://dx.doi.org/10.1093/jhmas/jrj038

[5] HSS, Inc. (2003) Definitions Manual for All-Payer Severity-adjusted DRG (APS-DRGs ${ }^{\circledR}$ ) Assignment, Public Use Version 20.0, 2003. HSS, Inc. https://www.hcup-us.ahrq.gov

[6] CMS (2013) Medicare Learning Network: Acute Care Hospital Inpatient Prospective Payment System.

http://www.cms.gov/Outreach-and-Education/Medicare-Learning-Network-MLN/MLNPr oducts/downloads/AcutePaymtSysfctsht.pdf

[7] Averill, R.F., Muldoon, J.H., Vertrees, J.C., Goldfield, N.I., Mullin, R.L., Fineran, E.C., Zhang, M.Z., Steinbeck, B. and Thelma, G.T. (1998) The Evolution of Casemix Measurement Using Diagnosis Related Groups (DRGs), 3M HIS Research Report 5-98.

[8] Wynn, B., Beckett, M., Hillborne, L., Scott, M. and Bahney, B. (2007) Evaluation of Severity-Adjusted DRG Systems: Interim Report, Prepared for the Centers for Medicare and Medicaid Services, released by Rand Health. 
[9] Leary, R.S., Mary, E., Johantgen, M.E., Farley, D., Forthman, M.T. and Wooster, D. (1997) All-Payer Severity-Adjusted Diagnosis-Related Groups: A Uniform Method to SeverityAdjust Discharge Data. Top Health Inform Manage, 17, 60-71. Aspen Publishers, Inc.

[10] Davis, M.P., Walsh, D., LeGrand, S.B., Lagman, R.L., Harrison, B. and Rybicki, L. (2004) The Financial Benefits of Acute Inpatient Palliative Medicine: An Inter-Institutional Comparative Analysis by All Patient Refined-Diagnosis Related Group and Case Mix Index. The Journal of Supportive Oncology, 3, 313-316.

[11] Lagman, R.L., Walsh, D., Davis, M.P. and Young, B. (2007) All Patient Refined-Diagnostic Related Group and Case Mix Index in Acute Care Palliative Medicine. Journal of Supportive Oncology, 5, 145-149.

[12] Horn, D.S., Sharkey, D.P., Chambers, A. and Horn, A.R. (1986) Severity of Illness within DRGs: Impact on Prospective Payment. American Journal of Public Health, 75, 1195-1199. http://dx.doi.org/10.2105/AJPH.75.10.1195

[13] Averill, R.F., McGuire, T.E., Manning, B.E., Fowler, D.A., Horn, S.D., Dickson, P.S., Coye, M.J., Knowlton, D.L. and Bender, J.A. (1992) A Study of the Relationship between Severity of Illness And Hospital Cost in New Jersey Hospitals. Health Services Research, 27, 587606.

[14] European Observatory on Health Care Systems (2016) HiT Countries Summaries. http://www.euro.who.int/en/about-us/partners/observatory/publications/health-system-rev iews-hits/full-list-of-country-hits

[15] Lungen, M., Dredge, B., Rose, A., Roebuck, C., Plamper, E. and Lauterbach, K. (2004) Using Diagnosis-Related Groups. The Situation in the United Kingdom National Health Service and in Germany. European Journal of Health Economics, 49, 287-289. http://dx.doi.org/10.1007/s10198-004-0267-9

[16] Wiley, M.M. (2014) Diagnosis Related Groups (DRGs): Measuring Hospital Case Mix.

[17] McKee, M. and Healy, J. (2002) Hospitals in a Changing Europe. Open University Press, Maidenhead.

[18] Huitfeldt, I.M.S., Häkkinen, U., Kruse, M., Medin, E., Rehnberg, C. and Rättö, H. (2015) Costs and Quality at the Hospital Level in the Nordic Countries. Health Economics, 24, 140-163. http://dx.doi.org/10.1002/hec.3260

[19] Paul, T. (2002) Paying Hospitals in Esthonia: Moving towards Needs Based Planning and Providing the Right Incentives. Estonian Health Insurance Fund. http://www.eurasiahealthtransitionconference.org/presenteng/PaluEng.pdf

[20] Busse, R., et al. (2002) Health Care Systems in Eight Countries: Trends and Challenges. European Observatory on Health Care Systems, London School of Economics \& Political Science Hub, London.

[21] Klein-Hitpaßa, U. and Scheller-Kreinsen, D. (2015) Policy Trends and Reforms in the German DRG-Based Hospital Payment System. Health Policy, 119, 252-257.

http://dx.doi.org/10.1016/j.healthpol.2015.01.006

[22] Busse, R. (2000) Germany Opts for Australian Diagnosis-Related Groups. Euro Observer, 2, 1-3.

[23] European Observatory on Health Care Systems (2010) Greece: Health System Review. Health Systems in Transition, 12, 1-180. http://www.euro.who.int/ data/assets/pdf file/0004/130729/e94660.pdf

[24] MoH (2011) Report on DRGs Implementation Study in the Greek NHS Hospitals. Ministry of Health. www.moh.gov.gr/articles/ken 
[25] Polyzos, N., Karanikas, H., Thireos, E., Kastanioti, C. and Kontodimopoulos, N. (2013) Reforming Reimbursement of Public Hospitals in Greece during the Economic Crisis: Implementation of a DRG System. Health Policy, 109, 14-22.

http://dx.doi.org/10.1016/j.healthpol.2012.09.011

[26] Schreyögg, J., Stargardt, T., Tiemann, O. and Busse, R. (2006) Methods to Determine Reimbursement Rates for Diagnosis Related Groups (DRG): A Comparison of Nine European Countries. Health Care Management Science, 9, 215-223.

http://dx.doi.org/10.1007/s10729-006-9040-1

[27] Fontain, P. (2004) Using Severity Adjustment Classification for Hospital Internal and External Benchmarking. IFHRO Congress \& AHIMA Convention Proceedings, Washington DC, October 2004. http://bok.ahima.org/doc?oid=59268\#.V7nneU2LTDc

[28] 3M (1998) All Patient Refined Diagnosis Related Groups Version 15.0 Definitions Manual. 1, 3M Health Information Systems.

[29] Goldfield, N. (1999) Physician Profiling and Risk Adjustment. Aspen Publishers, Gaithersburg, 413.

[30] Lorenzoni, L. (2000) Use of APR-DRGs in 15 Italian Hospitals. Case-Mix, 2, 131-135.

[31] Medpac (2015) Hospital Acute Inpatient Services Payment System. Medpac, Washington DC.

[32] Van de Voorde, C., Van den Heede, K., Obyn, C., Quentin, W., Geissler, A., Wittenbecher, F., Busse. R., Magnussen, J., Camaly, O., Devriese, S., Gerkens, S., Misplon, S., Neyt, M. and Mertens, R. (2014) Conceptual Framework for the Reform of the Belgian Hospital Payment System. Health Services Research (HSR), Belgian Health Care Knowledge Centre (KCE), KCE Reports 229, Brussels.

[33] Van de Voorde, C., Gerkens, S., Van den Heede, K. and Swartenbroekx, N. (2013) A Comparative Analysis of Hospital Care Payments in Five Countries. Health Services Research (HSR), Belgian Health Care Knowledge Centre (KCE), KCE Reports 207, Brussels.

[34] Hughes, J.S., Iezzoni, L.I., Daley, J. and Greenberg, L. (1996) How Severity Measures Rate Hospitalized Patients. Journal of General Internal Medicine, 11, 303-311. http://dx.doi.org/10.1007/BF02598273

[35] Iezzoni, L.I., Shwartz, M., Ash, A.S. and Mackiernan, Y.D. (1996) Does Severity Explain Differences in Hospital Length of Stay for Pneumonia Patients? Journal of Health Services Research \& Policy, 1, 65-76.

[36] Pirson, M., Delo, C., Di Pierdomenico, L., Laport, N., Biloque, V. and Leclercq, P. (2013) Variability of Nursing Care by APR-DRG and by Severity of Illness in a Sample of Nine Belgian Hospitals. BMC Nursing, 12, 26. http://dx.doi.org/10.1186/1472-6955-12-26

[37] Rubenstein, D., Friedman, G. and Bateman, D. (2013) RVUs and DRGs: Do They Fairly Reimburse Physicians and Hospitals and Incentivize Improved Care? Pediatrics, 131, 340342. http://dx.doi.org/10.1542/peds.2012-1434 
Annex

Code description

Economic Compensation $<$ Clinical Severity (large deviation).

\begin{tabular}{|c|c|}
\hline CODES & DESCRIPTION \\
\hline & Major surgeries for breast diseases \\
\hline D06A & Inflections/inflammations of the respiratory system with devastating systemic co-existing \\
\hline $\mathrm{A} 22 \mathrm{Ma}$ & diseases/complications \\
\hline TH06X & Thyroid surgery without damaging (systemic)or severe co-existing diseases-complications \\
\hline $\mathrm{A} 22 \mathrm{Mb}$ & Infections/inflammations of the respiratory tract with severe or moderate co-existing \\
\hline $\mathrm{K} 42 \mathrm{M}$ & conditions_complications \\
\hline \multirow[t]{3}{*}{ TH24M } & Heart failure and shock with disastrous (systemic) co-existing diseases-complications \\
\hline & Endocrine disorders with devastating (systemic) or severe co-existing diseases-complications \\
\hline & Sinus surgery and complex middle ear surgery. \\
\hline $\begin{array}{l}\text { W06A } \\
\text { Y04X }\end{array}$ & $\begin{array}{l}\text { Kidney operations, urinal operations and major procedures of the urinary bladder, for } \\
\text { non-neoplasmatic diseases without damaging (systemic) or severe co-existing medical } \\
\text { conditions-complications }\end{array}$ \\
\hline $\mathrm{N} 30 \mathrm{Mb}$ & $\begin{array}{l}\text { Stroke and other disorders of the cerebral vessels with severe co-existing diseases } \\
\text { complications. }\end{array}$ \\
\hline K46M & $\begin{array}{l}\text { Arrhythmia, cardiac arrest, and conduction disorders with devastating (systemic) or severe } \\
\text { co-existing diseases-complications }\end{array}$ \\
\hline
\end{tabular}

Economic Compensation $<$ Clinical Severity (small deviation).

\begin{tabular}{|c|c|}
\hline CODES & DESCRIPTION \\
\hline L01X & $\begin{array}{l}\text { Labor by caesarean section without damaging (systemic) or severe co-existing diseases } \\
\text { complications }\end{array}$ \\
\hline L02X & $\begin{array}{l}\text { Surgical operation of vaginal labor without damaging (systemic) or severe co-existing diseases } \\
\text { complications }\end{array}$ \\
\hline M03X & Hip replacement surgery without devastating (systemic) co-existing diseases-complications \\
\hline $\mathrm{P} 10 \mathrm{X}$ & Hernia surgery (inguinal, umbilical hernia, etc) without co-existing illnesses-complications 1 \\
\hline H08X & $\begin{array}{l}\text { Laparoscopic cholecystectomy with closed bile duct or catastrophic (systemic) or severe } \\
\text { co- existing illnesses-complications }\end{array}$ \\
\hline N03X & $\begin{array}{l}\text { Spine surgeries without damaging (systemic) or severe co-existing medical conditions- } \\
\text { complications }\end{array}$ \\
\hline Y20Ma & Kidney failure with devastating (systemic) co-existing diseases-complications \\
\hline Y23M & $\begin{array}{l}\text { Kidney and urinary system infections with devastating (systemic) or severe co-existing } \\
\text { diseases-complications }\end{array}$ \\
\hline W10A & Nasal Surgery \\
\hline
\end{tabular}


Economic Compensation >Clinical Severity (small deviation).

\begin{tabular}{|c|c|}
\hline CODES & DESCRIPTION \\
\hline M02X & $\begin{array}{l}\text { Skin graft without damaging (systemic) or severe co-existing medical conditions- } \\
\text { complications, with the exception of forearm }\end{array}$ \\
\hline $\mathrm{N} 23 \mathrm{~A}$ & $\begin{array}{l}\text { Dementia (deterioration of mental faculties) and other chronic dysfunction of cerebral } \\
\text { function }\end{array}$ \\
\hline K01X & $\begin{array}{l}\text { Implantation of automatic implantable cardiac defibrillator, complete system, without } \\
\text { catastrophic (systemic) co-existing diseases-complications }\end{array}$ \\
\hline M73M & $\begin{array}{l}\text { Post-operative monitoring musculoskeletal implants/prosthetics with devastating (systemic) or } \\
\text { severe co-existing diseases-complications }\end{array}$ \\
\hline $\mathrm{P} 04 \mathrm{Ma}$ & Lysis of peritoneal adhesions with devastating (systemic) co-existing diseases-complications \\
\hline T06X & Major surgery in neonate with admission weight $>2499 \mathrm{gr}$ with multiple major problems \\
\hline M07A & Amputation \\
\hline $\begin{array}{c}\text { N42Ma } \\
\text { D01X } \\
\text { TH01M }\end{array}$ & $\begin{array}{l}\text { Chronic and unspecified paraplegia/quadriplegia with or without surgical intervention with } \\
\text { disastrous (systemic) co-existing diseases-complications } \\
\text { Micro vascular tissue transfer for diseases, subcutaneous tissue and breast tissue without } \\
\text { damaging (systemic) or severe co-existing medical conditions-complications } \\
\text { Surgical operations for complications of diabetes with disastrous (systemic) co-existing } \\
\text { illnesses-complications }\end{array}$ \\
\hline
\end{tabular}

Submit or recommend next manuscript to SCIRP and we will provide best service for you:

Accepting pre-submission inquiries through Email, Facebook, LinkedIn, Twitter, etc. A wide selection of journals (inclusive of 9 subjects, more than 200 journals)

Providing 24-hour high-quality service

User-friendly online submission system

Fair and swift peer-review system

Efficient typesetting and proofreading procedure

Display of the result of downloads and visits, as well as the number of cited articles Maximum dissemination of your research work

Submit your manuscript at: http://papersubmission.scirp.org/

Or contact me@scirp.org 